\title{
Intestinal Microbial Metabolism of Phosphatidylcholine and Cardiovascular Risk
}

\author{
W.H. Wilson Tang, M.D., Zeneng Wang, Ph.D., Bruce S. Levison, Ph.D., Robert A. Koeth, \\ B.S., Earl B. Britt, M.D., Xiaoming Fu, M.S., Yuping Wu, Ph.D., and Stanley L. Hazen, M.D., \\ Ph.D. \\ Department of Cellular and Molecular Medicine, Lerner Research Institute, Cleveland Clinic, \\ Cleveland
}

\begin{abstract}
BACKGROUND—Recent studies in animals have shown a mechanistic link between intestinal microbial metabolism of the choline moiety in dietary phosphatidylcholine (lecithin) and coronary artery disease through the production of a proatherosclerotic metabolite, trimethylamine- $N$-oxide (TMAO). We investigated the relationship among intestinal microbiota-dependent metabolism of dietary phosphatidylcholine, TMAO levels, and adverse cardiovascular events in humans.
\end{abstract}

METHODS-We quantified plasma and urinary levels of TMAO and plasma choline and betaine levels by means of liquid chromatography and online tandem mass spectrometry after a phosphatidylcholine challenge (ingestion of two hard-boiled eggs and deuterium [d9]-labeled phosphatidylcholine) in healthy participants before and after the suppression of intestinal microbiota with oral broad-spectrum antibiotics. We further examined the relationship between fasting plasma levels of TMAO and incident major adverse cardiovascular events (death, myocardial infarction, or stroke) during 3 years of follow-up in 4007 patients undergoing elective coronary angiography.

RESULTS-Time-dependent increases in levels of both TMAO and its d9 isotopologue, as well as other choline metabolites, were detected after the phosphatidylcholine challenge. Plasma levels of TMAO were markedly suppressed after the administration of antibiotics and then reappeared after withdrawal of antibiotics. Increased plasma levels of TMAO were associated with an increased risk of a major adverse cardiovascular event (hazard ratio for highest vs. lowest TMAO quartile, 2.54; 95\% confidence interval, 1.96 to 3.28; $\mathrm{P}<0.001$ ). An elevated TMAO level predicted an increased risk of major adverse cardiovascular events after adjustment for traditional risk factors $(\mathrm{P}<0.001)$, as well as in lower-risk subgroups.

CONCLUSIONS-The production of TMAO from dietary phosphatidylcholine is dependent on metabolism by the intestinal microbiota. Increased TMAO levels are associated with an increased risk of incident major adverse cardiovascular events. (Funded by the National Institutes of Health and others.)

The phospholipid phosphatidylcholine (lecithin) is the major dietary source of choline, a semiessential nutrient that is part of the B-complex vitamin family. ${ }^{1,2}$ Choline has various metabolic roles, ranging from its essential involvement in lipid metabolism and cellmembrane structure to its role as a precursor for the synthesis of the neurotransmitter acetylcholine. Choline and some of its metabolites, such as betaine, can also serve as a

Copyright @ 2013 Massachusetts Medical Society

Address reprint requests to Dr. Hazen at the Cleveland Clinic, 9500 Euclid Ave. NC-10, Cleveland, OH 44195, or at hazens@ccf.org.

Disclosure forms provided by the authors are available with the full text of this article at NEJM.org. 
source of methyl groups that are required for proper metabolism of certain amino acids, such as homocysteine and methionine. ${ }^{3}$

There is a growing awareness that intestinal microbial organisms, collectively termed microbiota, participate in the global metabolism of their host. ${ }^{4-6}$ We recently described the potential role of a complex phosphatidylcholine-choline metabolic pathway involving gut microbiota in contributing to the pathogenesis of atherosclerotic coronary artery disease in animal models. ${ }^{7} \mathrm{We}$ also reported an association between a history of cardiovascular disease and elevated fasting plasma levels of trimethylamine- $N$-oxide (TMAO), an intestinal microbiota-dependent metabolite of the choline head group of phosphatidylcholine that is excreted in the urine. ${ }^{7-12}$

Here, we examine the relationship between oral intake of phosphatidylcholine and the involvement of the intestinal microbiota in the formation of TMAO in humans. We also examine the relationship between fasting plasma levels of TMAO and the long-term risk of incident major adverse cardiovascular events.

\section{METHODS \\ STUDY DESIGNS}

We designed and performed two prospective clinical studies, which were funded by the National Institutes of Health and approved by the institutional review board at the Cleveland Clinic. All participants provided written informed consent. In the first study

(phosphatidylcholine challenge), we enrolled 40 healthy adults who had no chronic illnesses (including a known history of heart, renal, pulmonary, or hematologic disease), had no active infections, and had not taken antibiotics or probiotics within the past month. Participants underwent a dietary phosphatidylcholine challenge during visit 1 (as described below). Six of these study participants were then given metronidazole (500 $\mathrm{mg}$ twice daily) plus ciprofloxacin (500 mg once daily) for 1 week. After receipt of the antibiotics, a repeat phosphatidylcholine challenge was performed at visit 2 . A third and final phosphatidylcholine challenge was performed 1 month or more after the withdrawal of antibiotics (and subsequent reacquisition of gut flora), at visit 3. After each challenge, choline metabolites were measured in plasma and urine, as described below.

In the second study (clinical outcomes), we enrolled 4007 adults who were undergoing elective diagnostic cardiac catheterization; the participants had no evidence of an acute coronary syndrome (cardiac troponin $\mathrm{T}$ level, $<0.1 \mu \mathrm{g}$ per liter, if available). A history of cardiovascular disease was defined as a documented history of coronary artery disease, peripheral artery disease, coronary or peripheral revascularization, stenosis of 50\% or more in one or more vessels observed during coronary angiography, or a remote history of either myocardial infarction or stroke. Fasting blood samples were obtained from all participants at the time of cardiac catheterization.

\section{LABORATORY TESTING}

Routine laboratory tests were performed, and samples were measured on the Abbott Architect platform (Abbott Laboratories), except for testing of myeloperoxidase, which was measured with the use of the CardioMPO test (Cleveland Heart Laboratories). Creatinine clearance was estimated with the use of the Cockcroft-Gault equation. TMAO was measured in plasma, as described below. Major adverse cardiovascular events (defined as death from any cause, nonfatal myocardial infarction, and nonfatal stroke) were ascertained and adjudicated for all participants during 3 years of follow-up. 


\section{PHOSPHATIDYLCHOLINE CHALLENGE}

A simple dietary phosphatidylcholine-choline challenge was administered to all participants in the first study. For each participant, baseline blood and spot urine samples were obtained after an overnight fast ( $\geq 12$ hours). At baseline, participants were given two large hardboiled eggs including yolk (containing approximately $250 \mathrm{mg}$ of total choline each) to be eaten within a 10-minute period together with $250 \mathrm{mg}$ of deuterium-labeled phosphatidylcholine (d9-phosphatidylcholine), as a tracer, contained in a gelatin capsule, which was administered under an investigational new drug exemption (IND number, 107940). Serial venous blood sampling was performed at 1, 2, 3, 4, 6, and 8 hours after baseline, along with a 24-hour urine collection.

The high-purity $\mathrm{d} 9$-phosphatidylcholine ( $>98 \%$ isotope enrichment) that was provided was synthesized from 1-palmitoyl-2-palmitoyl-sn-glycero-3-phosphoethanolamine after exhaustive methylation with d3-methyliodide (Cambridge Isotopes Laboratories). The d9phosphatidylcholine was isolated by means of sequential preparative thin-layer chromatography and high-performance liquid chromatography and was crystallized and dried under vacuum. Its purity (>99\%) was confirmed by both multinuclear nuclear magnetic resonance spectroscopy and mass spectrometry.

\section{MEASUREMENTS OF CHOLINE METABOLITES}

Plasma aliquots were isolated from whole blood collected in EDTA tubes, maintained at 0 to $4^{\circ} \mathrm{C}$ until processing within 4 hours and stored at $-80^{\circ} \mathrm{C}$. An aliquot from each 24-hour urine collection was spun to precipitate any potential cellular debris, and supernatants were stored at $-80^{\circ} \mathrm{C}$ until analysis. TMAO, trimethylamine, choline, betaine, and their $\mathrm{d} 9$ isotopologues were quantified with the use of a stable-isotope-dilution assay and highperformance liquid chromatography with on line electrospray ionization tandem mass spectrometry on an AB SCIEX QTRAP 5500 mass spectrometer; d4(1,1,2,2)-choline, d3 (methyl)-trimethylamine- $N$-oxide, and d3(methyl)-trimethylamine were used as internal standards. ${ }^{7}$ For measurement of trimethylamine in plasma, a sample aliquot was acidified to a final concentration of $60 \mathrm{mM}$ of hydrochloric acid before storage at $-80^{\circ} \mathrm{C}$. Levels of TMAO in urine were adjusted for urinary dilution by analysis of the urine creatinine level.

\section{STATISTICAL ANALYSIS}

In the clinical-outcomes study, we used Student's t-test and the Wilcoxon rank-sum test for continuous variables and the chi-square test for categorical variables to analyze the differences between participants who had major adverse cardiovascular events during follow-up and those who did not. For most analyses of outcomes, we divided plasma TMAO levels into quartiles. Where indicated, TMAO was also analyzed as a continuous variable, with the hazard ratio determined per standard-deviation change in the TMAO level. We used Kaplan-Meier analysis with Cox proportional-hazards regression for the time-to-event analysis to determine hazard ratios and $95 \%$ confidence intervals for major adverse cardiovascular events.

We performed Cox proportional-hazards regression analysis with adjustment for traditional cardiovascular risk factors (age, sex, systolic blood pressure, presence or absence of diabetes mellitus, low-density and high-density lipoprotein cholesterol levels, triglyceride levels, and smoking status) and for log-transformed high-sensitivity C-reactive protein levels (both alone and with myeloperoxidase), the log-transformed estimated glomerular filtration rate, total leukocyte count, body-mass index, medication history, and angiographic extent of coronary artery disease. For subgroup analyses, Cox proportional-hazards regression analysis was performed with adjustment for traditional cardiovascular risk factors and logtransformed high-sensitivity C-reactive protein levels. Improvement in model performance 
that was introduced by the inclusion of TMAO levels was evaluated with the use of net reclassification improvement. We calculated the $\mathrm{C}$ statistic using the area under the receiveroperating-characteristic (ROC) curve. Three-year predicted probabilities of a major adverse cardiovascular event were estimated from the Cox model. All analyses were performed with the use of $\mathrm{R}$ software, versions 2.8.0 and 2.15.1. A two-sided $\mathrm{P}$ value of less than 0.05 was considered to indicate statistical significance.

\section{RESULTS \\ PHOSPHATIDYLCHOLINE CHALLENGE}

For the 40 participants in the phosphatidylcholine challenge, plasma levels of TMAO are shown in Figure 1, and plasma levels of choline and betaine in Figure S1 in the Supplementary Appendix (available with the full text of this article at NEJM.org). Endogenous (unlabeled) TMAO (Fig. 1C) and choline and betaine (Fig. S1 in the Supplementary Appendix) were present in fasting plasma samples at baseline. Both TMAO and d9-TMAO were readily detected in plasma after the dietary phosphatidylcholine challenge at visit 1 (Fig. 1A and 1B). Time-dependent increases in the levels of both TMAO (Fig. 1C) and d9-TMAO (Fig. 1D) were also observed postprandially. Examination of 24hour urine specimens after the phosphatidylcholine challenge also showed the presence of both TMAO and d9-TMAO (Fig. S2 in the Supplementary Appendix). A strong correlation was observed between plasma levels of TMAO and the absolute urine TMAO level (Spearman's $r=0.58, \mathrm{P}<0.001$ ) and the urinary ratio of TMAO to creatinine (Spearman's $r$ $=0.91, \mathrm{P}<0.001)$. Time-dependent increases in the plasma levels of both the natural isotopes and d9-tracer forms of choline and betaine also increased after the phosphatidylcholine challenge (Fig. S1A and S1B in the Supplementary Appendix).

The suppression of intestinal microbiota by the administration of oral broad-spectrum antibiotics for 1 week in six of the participants resulted in near-complete suppression of detectable TMAO and d9-TMAO after the phosphatidylcholine challenge during visit 2 in both plasma (Fig. 1) and urine (Fig. S2 in the Supplementary Appendix). In parallel analyses, postprandial elevations in plasma trimethlyamine and $\mathrm{d} 9$-trimethylamine levels were observed after the phosphatidylcholine challenge, but the levels were undetectable after the administration of antibiotics (data not shown). In contrast, the time courses for postprandial changes in free choline and betaine (natural isotopes and d9-isotopologues) were not altered by suppression of intestinal micro-biota (Fig. S1 in the Supplementary Appendix).

After the withdrawal of antibiotics and subsequent reappearance of intestinal microbiota over a period of 1 month or longer, the phosphatidylcholine challenge during visit 3 again resulted in readily detectable and time-dependent changes in TMAO and d9-TMAO in plasma (Fig. 1) and urine (Fig. S2 in the Supplementary Appendix). The extent to which TMAO levels in plasma at visit 3 returned to preantibiotic levels was variable, a finding that is consistent with reports describing variable recovery of intestinal microbiota after antibiotic cessation. ${ }^{13,14}$

\section{CLINICAL OUTCOMES}

TMAO Levels and Cardiovascular Events-The baseline characteristics of the 4007 participants in the clinical-outcomes study are shown in Table 1, according to whether they had a major adverse cardiovascular event during the 3-year follow-up. The mean age of the participants was 63 years, and two thirds were men; the prevalence of cardiovascular risk factors was high, and many of the participants had at least single-vessel coronary disease. Participants who had incident major adverse cardiovascular events during 3 years of follow- 
up had higher risk profiles at baseline than those without events, including an older age, higher fasting glucose levels, and higher rates of diabetes, hypertension, and previous myocardial infarction.

Participants who had major adverse cardiovascular events also had higher baseline levels of TMAO, as compared with those who did not have cardiovascular events (median, $5.0 \mu \mathrm{M}$ [interquartile range, 3.0 to 8.8 ] vs. $3.5 \mu \mathrm{M}$ [interquartile range, 2.4 to 5.9]; $\mathrm{P}<0.001$ ) (Table 1). As compared with participants in the lowest quartile of TMAO levels, those in the highest quartile had a significantly increased risk of an event (hazard ratio, 2.54; $95 \%$ confidence interval $[\mathrm{CI}], 1.96$ to $3.28 ; \mathrm{P}<0.001$ ) (Table 2).

After adjustment for traditional risk factors and other baseline covariates, elevated plasma levels of TMAO remained a significant predictor of the risk of major adverse cardiovascular events (Table 2). We observed a graded increase in risk with increasing levels of TMAO, as illustrated in the Kaplan-Meier analysis shown in Figure 2. A similar graded increase in risk was observed when levels of TMAO were analyzed as a continuous variable in increments of $1 \mathrm{SD}$ (unadjusted hazard ratio, 1.40 [95\% CI, 1.29 to 1.51; $\mathrm{P}<0.001$ ]; adjusted hazard ratio, 1.30 [95\% CI, 1.20 to $1.41 ; \mathrm{P}<0.001])$.

When the components of the major adverse cardiovascular events were analyzed separately, increased levels of TMAO remained significantly associated with an increased risk of death (hazard ratio, 3.37; 95\% CI, 2.39 to $4.75 ; \mathrm{P}<0.001$ ) and nonfatal myocardial infarction or stroke (hazard ratio, $2.13 ; 95 \% \mathrm{CI}, 1.48$ to $3.05 ; \mathrm{P}<0.001$ ). The inclusion of TMAO as a covariate resulted in a significant improvement in risk estimation over traditional risk factors (net reclassification improvement, $8.6 \%[\mathrm{P}<0.001]$; integrated discrimination improvement, $9.2 \%$ [P<0.001]; $\mathrm{C}$ statistic, $68.3 \%$ vs. $66.4 \%[\mathrm{P}=0.01]$ ). In a separate analysis, we excluded all participants who underwent revascularization within 30 days after enrollment in the study. In this subcohort of 3475 participants, TMAO remained significantly associated with the risk of major adverse cardiovascular events (unadjusted hazard ratio for highest quartile vs. lowest quartile, 2.47 [95\% CI, 1.87 to 3.27]; $\mathrm{P}<0.001$ ).

Cardiovascular Risk in Low-Risk Subgroups-The prognostic value of elevated plasma levels of TMAO for cardiovascular risk remained significant in various subgroups associated with a reduced overall risk of major adverse cardiovascular events (Fig. S3 in the Supplementary Appendix). These subgroups included younger participants ( $<65$ years of age), women, and participants who did not have a known history of coronary artery disease or coronary disease risk equivalents, had low lipid and apolipoprotein levels, had normal blood pressure, did not smoke, and had low levels of other known risk markers, such as Creactive protein, myeloperoxidase, and white-cell count.

\section{DISCUSSION}

Studies in germ-free mice and cross-sectional clinical studies in humans have suggested a role for the intestinal microbiota in the pathogenesis of atherosclerosis in patients with a diet rich in phosphatidylcholine (with major sources including eggs, liver, beef, and pork) through the formation of the metabolite trimethylamine and conversion to TMAO. ${ }^{7,15}$ In our study, we describe the generation of the proatherogenic metabolite TMAO from dietary phosphatidylcholine through the use of stable-isotope-tracer feeding studies. We further found a role for the intestinal microbiota in the production of TMAO through its suppression by means of oral broad-spectrum antibiotics and then reacquisition of trimethylamine and the production of TMAO from dietary phosphatidylcholine after the withdrawal of antibiotics and subsequent intestinal recolonization. Finally, we describe the potential clinical significance of this intestinal microbiota-dependent metabolite by showing that 
fasting plasma TMAO levels predict the risk of incident major adverse cardiovascular events independently of traditional cardiovascular risk factors and the presence or extent of coronary artery disease and within multiple low-risk subgroups, including both participants without angiographic evidence of substantial coronary artery disease (i.e., stenosis of $<50 \%$ in major coronary vessels) and those with low-risk lipid and apolipoprotein levels. Our findings suggest that pathways that are dependent on the intestinal microbiota may contribute to the pathophysiology of atherosclerotic coronary artery disease and suggest potential therapeutic targets.

The intestinal microbiota have previously been implicated in complex metabolic diseases such as obesity. ${ }^{4-6,16-18}$ However, the involvement of microbiota in the inception of atherosclerosis in humans has only recently been suggested. ${ }^{7}$ The ability of oral broadspectrum antibiotics to temporarily suppress the production of TMAO is a direct demonstration that intestinal microorganisms play an obligatory role in the production of TMAO from phosphatidylcholine in humans. Intestinal microbiota convert the choline moiety of dietary phosphatidylcholine into trimethylamine, which is subsequently converted into TMAO by hepatic flavin-containing monooxygenases (Fig. 3) ${ }^{20-23}$ The observed delay in the detection of plasma d9-TMAO levels after the ingestion of d9-phosphatidylcholine may reflect the time required for the conversion of trimethylamine into TMAO ${ }^{24}$ since separate analyses monitoring trimethylamine and d9-trimethylamine production showed a time course consistent with a precursor-to-product relationship (data not shown). TMAO has been identified in fish as an important osmolite, ${ }^{25}$ and the ingestion of fish raises urinary TMAO levels. Nevertheless, the high correlation between urine and plasma levels of TMAO argues for effective urinary clearance of TMAO. Hence, an efficient excretion mechanism may provide protection by preventing the accumulation of TMAO and does not undermine the mechanistic link between TMAO and cardiovascular risk.

Although an association between infectious organisms and atherosclerosis has previously been postulated, studies looking at the role of antimicrobial therapy in preventing disease progression have been disappointing. ${ }^{26,27}$ It is important to recognize that the choice of antimicrobial therapy in previous intervention trials was based largely on targeting postulated organisms rather than modulating the composition of intestinal microbiota or their metabolites. Furthermore, even if an antibiotic initially suppressed TMAO levels, the durability of that effect with long-term use remains unknown. In unpublished studies, we observed that long-term use of a single antibiotic (6 months of ciprofloxacin), which initially suppressed plasma TMAO levels in a rodent model, completely lost its suppressive effect, an observation that is consistent with the expansion of antibiotic-resistant intestinal microbiota. Thus, instead of suggesting that intestinal microbes should be eradicated with long-term use of antibiotics, our findings point to the possibility that plasma TMAO levels may identify a pathway within intestinal microbiota amenable to therapeutic modulation. For example, our data suggest that excessive consumption of dietary phosphatidylcholine and choline should be avoided; a vegetarian or high-fiber diet can reduce total choline intake. ${ }^{16}$ It should also be noted that choline is a semiessential nutrient and should not be completely eliminated from the diet, since this can result in a deficiency state. However, standard dietary recommendations, if adopted, will limit the intake of phosphatidylcholineand cholinerich foods, since these foods are also typically high in fat and cholesterol content. ${ }^{1}$ An alternative potential therapeutic intervention is targeting the composition of the microbiota or biochemical pathways, with either a functional food such as a probiotic ${ }^{17}$ or a pharmacologic intervention. The latter hypothetically could take the form of treatment with an inhibitor to block specific microbial metabolic pathways or a short course of nonsystemic antibiotics to reduce the burden of TMAO-producing microbes, two therapies that have been used in the treatment of irritable bowel syndrome. ${ }^{28}$ Further studies are warranted to establish whether antimicrobial therapies can significantly reduce cardiovascular risk. 
In conclusion, we have shown that intestinal microbes participate in phosphatidylcholine metabolism to form circulating and urinary TMAO. We also established a correlation between high plasma levels of TMAO and an increased risk of incident major adverse cardiovascular events that is independent of traditional risk factors, even in low-risk cohorts.

\section{Supplementary Material}

Refer to Web version on PubMed Central for supplementary material.

\section{Acknowledgments}

Supported by grants from the National Institutes of Health and its Office of Dietary Supplements (R01HL103866 and 1P20HL113452). The clinical study GeneBank was supported by grants from the National Institutes of Health (P01HL098055, P01HL076491, R01HL103931, and R01DK080732) and a Cleveland Clinic/Case Western Reserve University Clinical and Translational Science Award (UL1TR000439). Dr. Hazen was supported by a gift from the Leonard Krieger Fund. Mass spectrometry instrumentation used was housed within the Cleveland Clinic Mass Spectrometry Facility with partial support through a Center of Innovation by AB SCIEX.

We thank Linda Kerchenski and Cindy Stevenson for their assistance in recruitment of study participants and Amber Gist and Naomi Bongorno for their assistance in the preparation of earlier versions of the figures and the manuscript.

\section{REFERENCES}

1. Patterson, KY.; Bhagwat, SA.; Williams, JR.; Howe, JC.; Holden, JM. USDA database for the choline content of common foods: release two. 2008. (http://www.ars.usda.gov/SP2UserFiles/Place/ 12354500/Data/Choline/Choln02.pdf)

2. Zhang AQ, Mitchell SC, Smith RL. Dietary precursors of trimethylamine in man: a pilot study. Food Chem Toxicol. 1999; 37:515-520. [PubMed: 10456680]

3. Zeisel SH. Choline: critical role during fetal development and dietary requirements in adults. Annu Rev Nutr. 2006; 26:229-250. [PubMed: 16848706]

4. Dumas ME, Barton RH, Toye A, et al. Metabolic profiling reveals a contribution of gut microbiota to fatty liver phenotype in insulin-resistant mice. Proc Natl Acad Sci U S A. 2006; 103:1251112516. [PubMed: 16895997]

5. Wen L, Ley RE, Volchkov PY, et al. Innate immunity and intestinal microbiota in the development of Type 1 diabetes. Nature. 2008; 455:1109-1113. [PubMed: 18806780]

6. Bäckhed F, Ding H, Wang T, et al. The gut microbiota as an environmental factor that regulates fat storage. Proc Natl Acad Sci U S A. 2004; 101:15718-15723. [PubMed: 15505215]

7. Wang Z, Klipfell E, Bennett BJ, et al. Gut flora metabolism of phosphatidylcholine promotes cardiovascular disease. Nature. 2011; 472:57-63. [PubMed: 21475195]

8. de la Huerga J, Popper H. Urinary excretion of choline metabolites following choline administration in normals and patients with hepatobiliary diseases. J Clin Invest. 1951; 30:463-470. [PubMed: 14832377]

9. Simenhoff ML, Saukkonen JJ, Burke JF, Wesson LG, Schaedler RW. Amine metabolism and the small bowel in uraemia. Lancet. 1976; 2:818-821. [PubMed: 61496]

10. Ihle BU, Cox RW, Dunn SR, Simenhoff ML. Determination of body burden of uremic toxins. Clin Nephrol. 1984; 22:82-89. [PubMed: 6383674]

11. Bain MA, Fornasini G, Evans AM. Trimethylamine: metabolic, pharmacokinetic and safety aspects. Curr Drug Metab. 2005; 6:227-240. [PubMed: 15975041]

12. Erdmann CC. On the alleged occurrence of trimethylamine in the urine. J Biol Chem. 1910; 8:5760.

13. Hooper LV, Gordon JI. Commensal host-bacterial relationships in the gut. Science. 2001; 292:1115-1118. [PubMed: 11352068]

14. Jernberg C, Löfmark S, Edlund C, Jansson JK. Long-term impacts of antibiotic exposure on the human intestinal microbiota. Microbiology. 2010; 156:3216-3223. [PubMed: 20705661] 
15. al-Waiz M, Mikov M, Mitchell SC, Smith RL. The exogenous origin of trimethylamine in the mouse. Metabolism. 1992; 41:135-136. [PubMed: 1736035]

16. Stella C, Beckwith-Hall B, Cloarec O, et al. Susceptibility of human metabolic phenotypes to dietary modulation. J Proteome Res. 2006; 5:2780-2788. [PubMed: 17022649]

17. Martin FP, Wang Y, Sprenger N, et al. Probiotic modulation of symbiotic gut microbial-host metabolic interactions in a humanized microbiome mouse model. Mol Syst Biol. 2008; 4:157. [PubMed: 18197175]

18. Gill SR, Pop M, Deboy RT, et al. Metagenomic analysis of the human distal gut microbiome. Science. 2006; 312:1355-1359. [PubMed: 16741115]

19. Loscalzo J. Lipid metabolism by gut microbes and atherosclerosis. Circ Res. 2011; 109:127-129. [PubMed: 21737814]

20. Bennett BJ, de Aguiar Vallim TQ, Wang Z, et al. Trimethylamine-N-oxide, a metabolite associated with atherosclerosis, exhibits complex genetic and dietary regulation. Cell Metab. 2013; 17:49-60. [PubMed: 23312283]

21. Möller B, Hippe H, Gottschalk G. Degradation of various amine compounds by mesophilic clostridia. Arch Microbiol. 1986; 145:85-90. [PubMed: 3753143]

22. Lang DH, Yeung CK, Peter RM, et al. Isoform specificity of trimethylamine N-oxygenation by human flavin-containing monooxygenase (FMO) and P450 enzymes: selective catalysis by FMO3. Biochem Pharmacol. 1998; 56:1005-1012. [PubMed: 9776311]

23. Bennett BJ, Vallim TQ, Wang Z, et al. Trimethylamine-N-oxide, a metabolite associated with atherosclerosis, exhibits complex genetic and dietary regulation. Cell Metab. 2013; 17:49-60. [PubMed: 23312283]

24. Al-Waiz M, Mitchell SC, Idle JR, Smith RL. The relative importance of N-oxidation and Ndemethylation in the metabolism of trimethylamine in man. Toxicology. 1987; 43:117-121. [PubMed: 3810655]

25. Yancey PH, Rhea MD, Kemp KM, Bailey DM. Trimethylamine oxide, betaine and other osmolytes in deep-sea animals: depth trends and effects on enzymes under hydrostatic pressure. Cell Mol Biol (Noisy-le-grand). 2004; 50:371-376. [PubMed: 15529747]

26. Cannon CP, Braunwald E, McCabe CH, et al. Antibiotic treatment of Chlamydia pneumoniae after acute coronary syndrome. N Engl J Med. 2005; 352:1646-1654. [PubMed: 15843667]

27. Grayston JT, Kronmal RA, Jackson LA, et al. Azithromycin for the secondary prevention of coronary events. N Engl J Med. 2005; 352:1637-1645. [PubMed: 15843666]

28. Pimentel M, Lembo A, Chey WD, et al. Rifaximin therapy for patients with irritable bowel syndrome without constipation. N Engl J Med. 2011; 364:22-32. [PubMed: 21208106] 


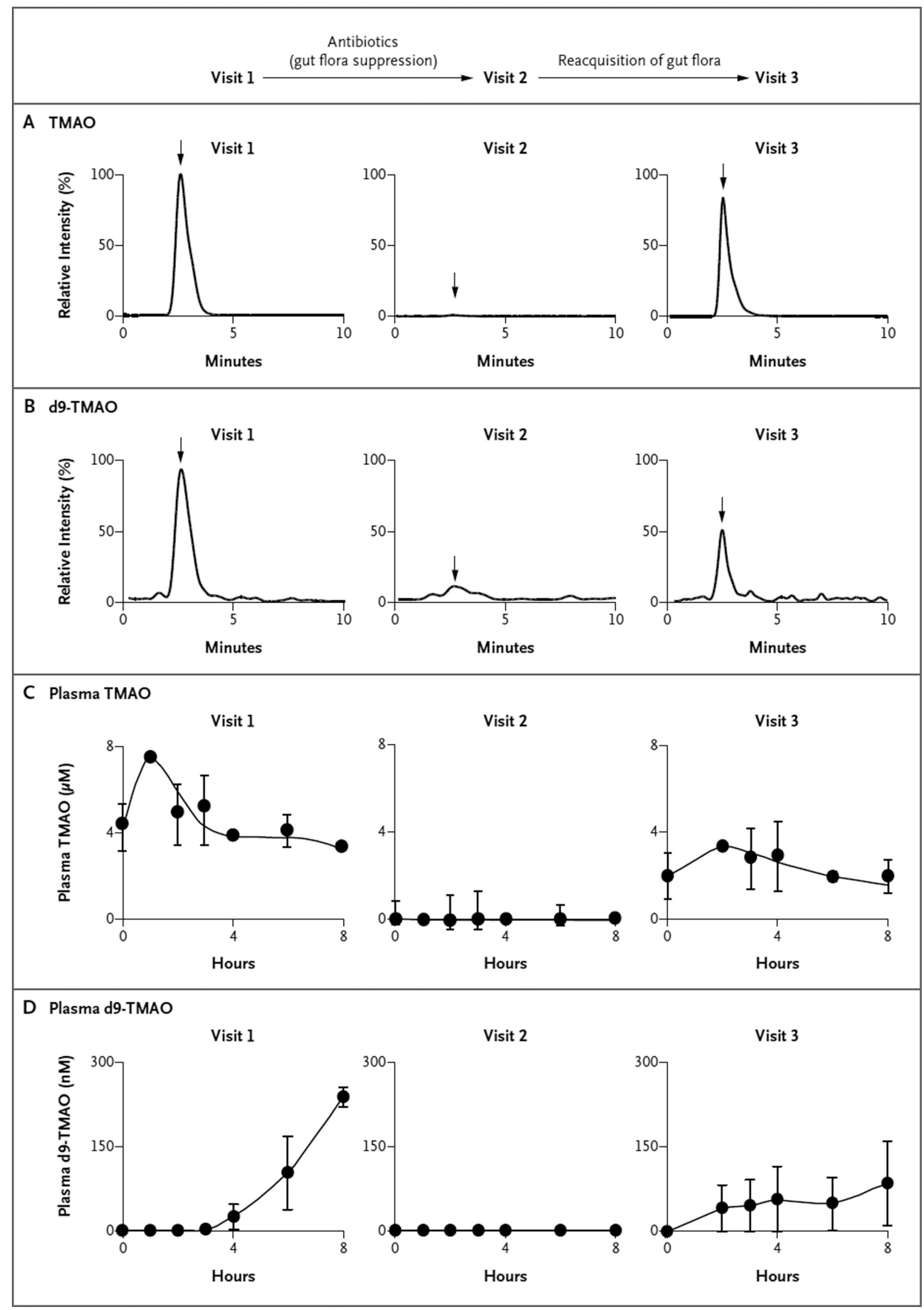

Figure 1. Effects of a Phosphatidylcholine Challenge and Administration of Antibiotics on Mean Levels of Trimethylamine- $\boldsymbol{N}$-Oxide (TMAO) and Its d9 Isotopologue (d9-TMAO)

All 40 study participants underwent the first dietary phosphatidylcholine challenge (visit 1), which consisted of the ingestion of deuterium-labeled phosphatidylcholine (d9phosphatidylcholine) and two hard-boiled eggs. Six participants then received broadspectrum antibiotics for 1 week, followed by a second phosphatidylcholine challenge (visit 2). These same participants returned again at least 1 month after discontinuing the antibiotics for a third challenge (visit 3). Shown are the results of assays for TMAO (Panel A) and d9-TMAO (Panel B) after the phosphatidylcholine challenge, before and after the administration of antibiotics, with the intensity of stable-isotope-dilution assays measured by 
means of high-performance liquid chromatography with online electro-spray ionization tandem mass spectrometry. The arrows indicate retention time where authentic isotopelabeled TMAO standards elute. Also shown are the plasma levels of TMAO (Panel C) and d9-TMAO (Panel D) at each visit. The plasma levels of TMAO were markedly suppressed after the administration of antibiotics and subsequently reappeared after the cessation of antibiotics, indicating that the production of TMAO from dietary phosphatidylcholine is dependent on metabolism by the intestinal microbiota. 


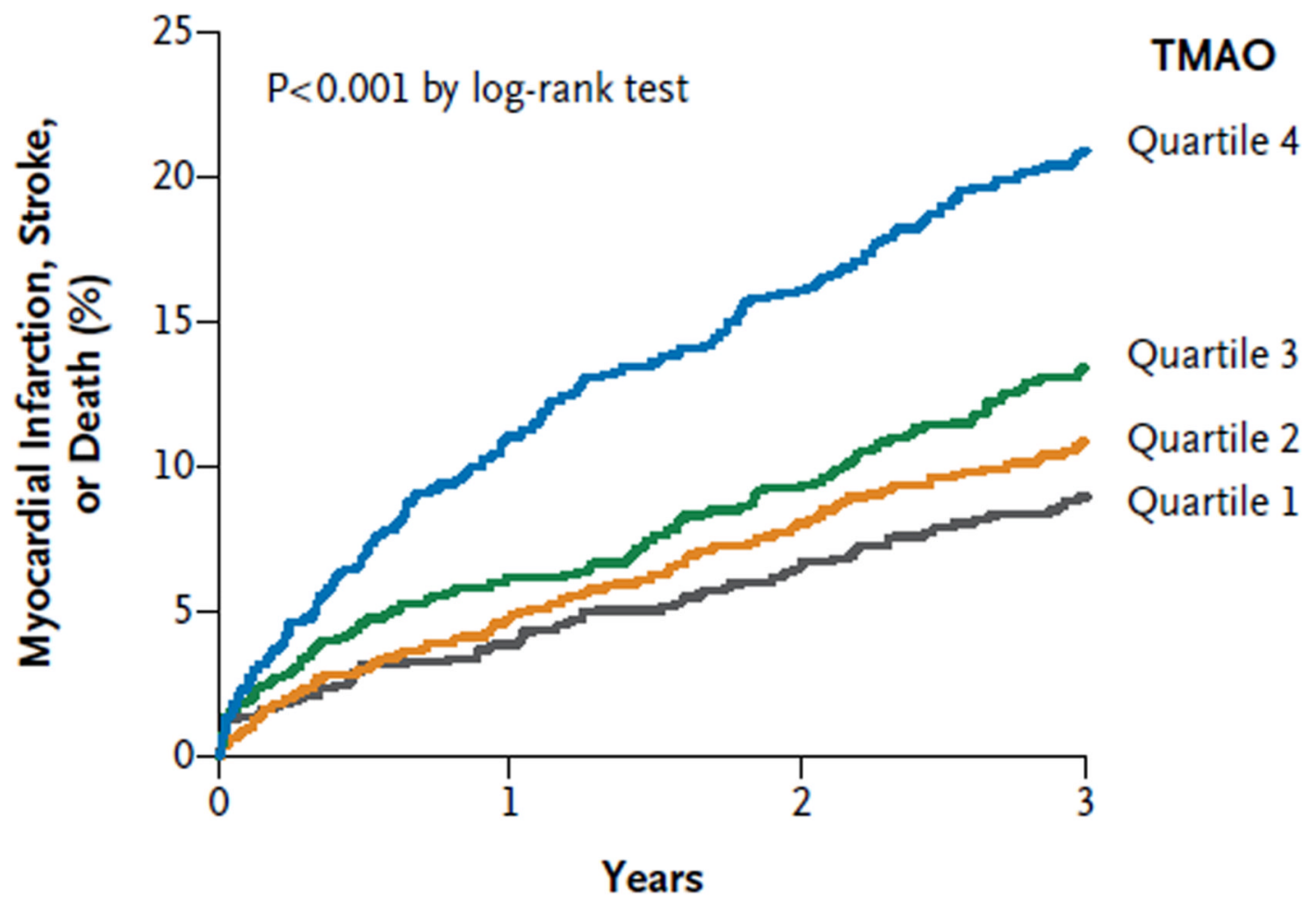

No. at Risk

Quartile 1

1001

933

869

827

Quartile 2

998

940

884

843

Quartile 3

1003

938

888

835

Quartile 4

913

849

791

Figure 2. Kaplan-Meier Estimates of Major Adverse Cardiovascular Events, According to the Quartile of TMAO Level

Data are shown for 4007 participants in the clinical-outcomes study. The $\mathrm{P}$ value is for all comparisons. 


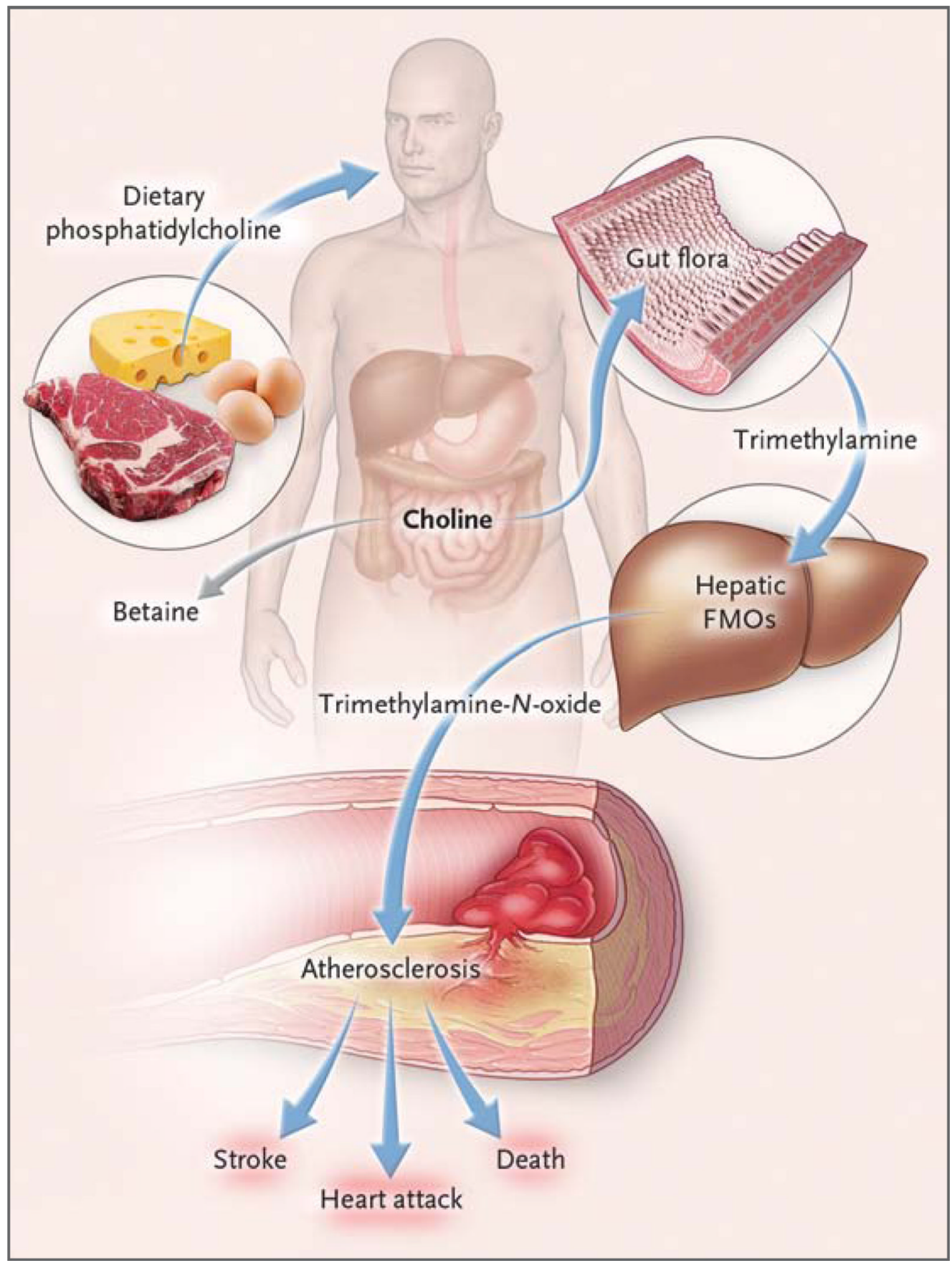

Figure 3. Pathways Linking Dietary Phosphatidylcholine, Intestinal Microbiota, and Incident Adverse Cardiovascular Events

Ingested phosphatidylcholine (lecithin), the major dietary source of total choline, is acted on by intestinal lipases to form a variety of metabolic products, including the cholinecontaining nutrients glycerophosphocholine, phosphocholine, and choline. Cholinecontaining nutrients that reach the cecum and large bowel may serve as fuel for intestinal microbiota (gut flora), producing trimethylamine (TMA). TMA is rapidly further oxidized to trimethylamine- $N$-oxide (TMAO) by hepatic flavin-containing monooxygenases (FMOs). TMAO enhances the accumulation of cholesterol in macro-phages, the accumulation of foam cells in artery walls, and atherosclerosis, ${ }^{7}$ all factors that are associated with an 
increased risk of heart attack, stroke, and death. Choline can also be oxidized to betaine in both the liver and kid-neys. ${ }^{20}$ Dietary betaine can serve as a substrate for bacteria to form $\mathrm{TMA}^{21}$ and presumably TMAO. 


\section{Table 1}

Baseline Characteristics of the Participants in the Clinical-Outcomes Study, According to Status with Respect to Major Adverse Cardiovascular Events at 3 Years. *

\begin{tabular}{|c|c|c|c|c|}
\hline Characteristic & $\begin{array}{l}\text { All Participants } \\
\qquad(\mathrm{N}=4007)\end{array}$ & $\begin{array}{l}\text { Participants } \\
\text { without Events } \\
(\mathrm{N}=\mathbf{3 4 9 4})\end{array}$ & $\begin{array}{l}\text { Participants } \\
\text { with Events } \\
(\mathbf{N}=\mathbf{5 1 3})\end{array}$ & P Value ${ }^{\dagger}$ \\
\hline Age $-\mathrm{yr}$ & $63 \pm 11$ & $62 \pm 11$ & $68 \pm 10$ & $<0.001$ \\
\hline Male sex - \% & 64 & 65 & 62 & 0.16 \\
\hline Median body-mass index (interquartile range) ${ }^{*}$ & $28.7(25.6-32.5)$ & $28.7(25.7-32.5)$ & $28.1(24.8-32.4)$ & 0.03 \\
\hline Diabetes mellitus — \% & 32 & 30 & 43 & $<0.001$ \\
\hline Hypertension - \% & 72 & 71 & 79 & $<0.001$ \\
\hline History of myocardial infarction - $\%$ & 42 & 40 & 53 & $<0.001$ \\
\hline \multicolumn{5}{|l|}{ No. of coronary vessels with $\geq 50 \%$ stenosis - $\%$} \\
\hline 0 & 26 & 28 & 15 & $<0.001$ \\
\hline 1 & 20 & 21 & 15 & 0.004 \\
\hline 2 & 20 & 20 & 21 & 0.51 \\
\hline 3 & 34 & 32 & 48 & $<0.001$ \\
\hline Current or former smoker - $\%$ & 65 & 65 & 69 & 0.05 \\
\hline \multicolumn{5}{|l|}{ Median cholesterol (interquartile range) $-\mathrm{mg} / \mathrm{dl}$} \\
\hline Low-density lipoprotein & $96(78-117)$ & $96(78-117)$ & $96(75-116)$ & 0.34 \\
\hline High-density lipoprotein & $34(28-41)$ & $34(28-41)$ & $33(28-40)$ & 0.03 \\
\hline Median triglycerides (interquartile range) $-\mathrm{mg} / \mathrm{dl}$ & $118(85-170)$ & $118(85-169)$ & $124(86-173)$ & 0.52 \\
\hline \multicolumn{5}{|l|}{ Median apolipoprotein (interquartile range) $-\mathrm{mg} / \mathrm{dl}$} \\
\hline $\mathrm{B}$ & $82(69-96)$ & $82(69-96)$ & $82(68-96)$ & 0.86 \\
\hline A1 & $116(103-133)$ & $117(103-133)$ & $114(100-129)$ & 0.002 \\
\hline Median fasting glucose (interquartile range) - mg/dl & $102(93-119)$ & $102(92-117)$ & $106(94-135)$ & $<0.001$ \\
\hline $\begin{array}{l}\text { Median high-sensitivity C-reactive protein (interquartile range) } \\
-\mathrm{ng} / \mathrm{liter}\end{array}$ & $2.4(1-5.9)$ & $2.3(1-5.5)$ & $3.9(1.8-9.8)$ & $<0.001$ \\
\hline Median myeloperoxidase (interquartile range) $-\mathrm{pM}$ & $115.2(76.4-245.7)$ & $113.2(75.4-238.3)$ & $136.3(84.7-329.3)$ & $<0.001$ \\
\hline $\begin{array}{l}\text { Median estimated glomerular filtration rate (interquartile } \\
\text { range) } \\
-\mathrm{ml} / \mathrm{min} / 1.73 \mathrm{~m}^{2}\end{array}$ & $82(69-95)$ & $83(71-96)$ & $75(56-89)$ & $<0.001$ \\
\hline Median white-cell count (interquartile range) - per $\mathrm{mm}^{3}$ & $6100(5100-7500)$ & $6100(5000-7500)$ & $6400(5300-8100)$ & 0.001 \\
\hline \multicolumn{5}{|l|}{ Medications - \% } \\
\hline Aspirin & 74 & 74 & 70 & 0.04 \\
\hline ACE inhibitor or ARB & 50 & 49 & 58 & $<0.001$ \\
\hline Statin & 60 & 61 & 56 & 0.06 \\
\hline Beta-blocker & 63 & 63 & 65 & 0.41 \\
\hline Median TMAO (interquartile range) $-\mu \mathrm{M}$ & $3.7(2.4-6.2)$ & $3.5(2.4-5.9)$ & $5.0(3.0-8.8)$ & $<0.001$ \\
\hline
\end{tabular}

* Plus-minus values are means \pm SD. To convert the values for cholesterol to millimoles per liter, multiply by 0.02586 . To convert the values for triglycerides to millimoles per liter, multiply by 0.01129 . To convert the values for glucose to millimoles per liter, multiply by 0.05551 . ACE denotes angiotensin-converting enzyme, ARB angiotensin-receptor blocker, and TMAO trimethylamine- $N$-oxide. 
${ }^{\dagger}$ The $\mathrm{P}$ value is for the comparison between patients who had a major adverse cardiovascular event and those who did not.

The body-mass index is the weight in kilograms divided by the square of the height in meters. 


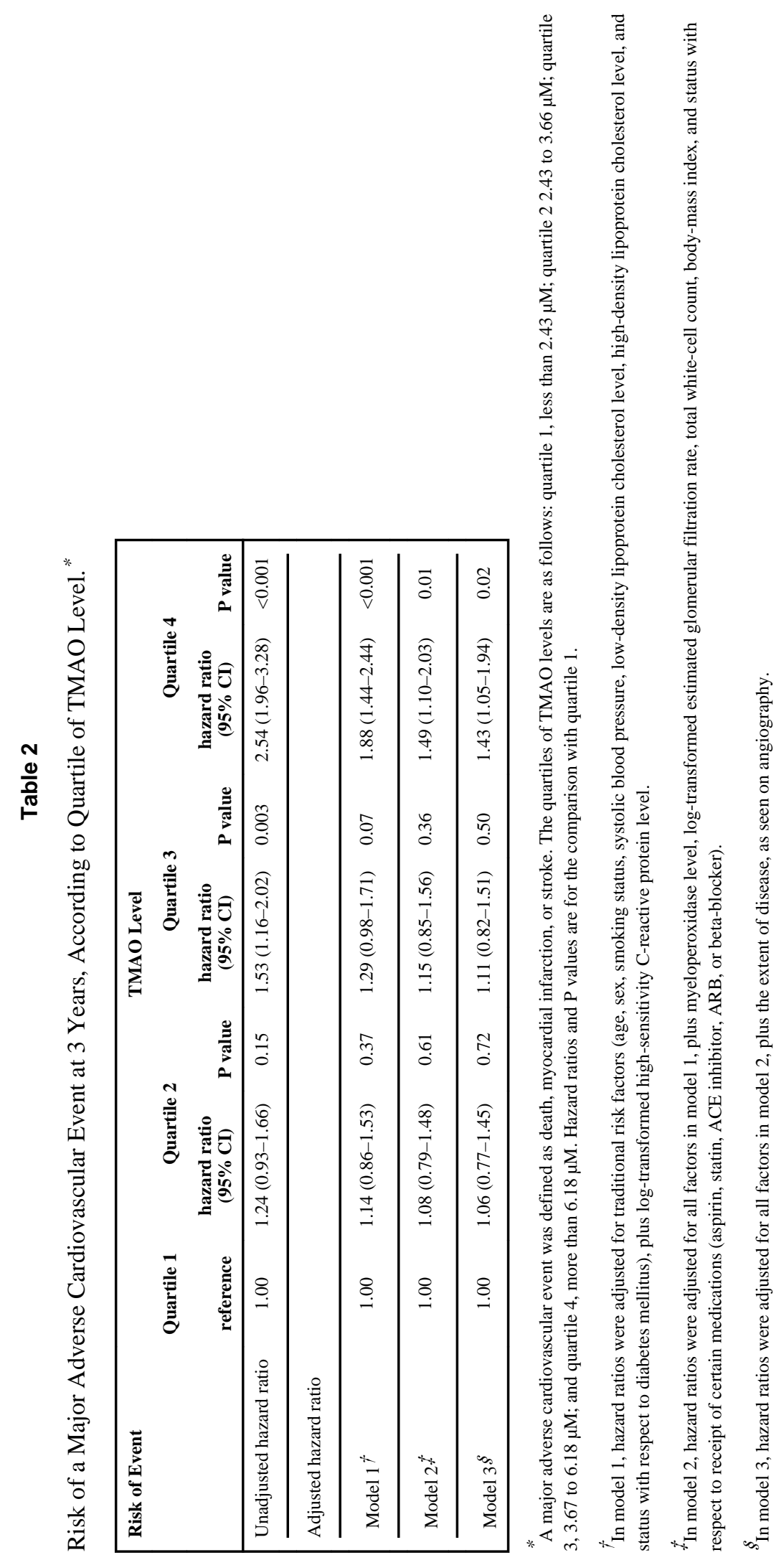

NEngl J Med. Author manuscript; available in PMC 2013 October 25. 